\title{
STABILIZING LINEAR MPC WITH EFFICIENT PRIORITIZED INFEASIBILITY HANDLING
}

\author{
Jostein Vada* Olav Slupphaug* Tor Arne Johansen* \\ Bjarne A. Foss ${ }^{*, 1}$ \\ * Department of Engineering Cybernetics \\ Norwegian University of Science and Technology, Norway
}

\begin{abstract}
In order to minimize the number of situations when a model predictive controller fails to compute a control input, all practical MPC implementations should have a means to recover from infeasibility. We present a recently developed infeasibility handler which computes optimal relaxations of the relaxable constraints subject to a user-defined prioritization. This infeasibility handler requires that only a single linear program needs to be solved on-line in addition to the standard quadratic programming problem. The method is illustrated on an example. Copyright 2000 IFAC
\end{abstract}

Keywords: Model based control, infeasibility handling, prioritization, linear systems.

\section{INTRODUCTION}

During the last years, model predictive control (MPC) has become an attractive control strategy within the process industries. Important stability results within the area of linear MPC are given in (Rawlings and Muske, 1993) under the assumption of feasibility. In order to fully exploit this stabilizing property, a means to recover from infeasibility of the associated optimization problem whenever possible is required. Note that in the MPC controller proposed by Rawlings and Muske (1993), an approach for handling infeasibilities caused by the state constraints is included. Infeasibility problems may occur due to e.g. disturbances, operator intervention, modelling errors, or plant failures.

Constraints representing physical limitations must be enforced at all times (non-relaxable). Other constraints should be satisfied whenever possible (relaxable), but may be relaxed when necessary. In order to transform an infeasible MPC optimization problem into a feasible one, there must exist a solution to the non-relaxable constraints. If no such solution exists, some alternative control strategy must be activated. Note that in a typical MPC implementation, there is a large number of constraints. When infeasibility occurs, it is often not obvious which constraints to relax and the amount that these constraints should be relaxed in order to render a consistent set of constraints.

In the literature, there are not many contributions on this practically important issue. The fol-

1 Corresponding author, email: Bjarne.Foss@itk.ntnu.no, address: Dept. of Eng. Cybernetics, NTNU, 7491 Trondheim, Norway, phone: +4773594476 , fax: +4773594399 lowing papers contain contributions to this field: (Rawlings and Muske, 1993), (Scokaert and Rawlings, 1999), (Garcia and Morshedi, 1986), (Qin and Badgwell, 1996), (Tyler and Morari, 1999), (Scokaert, 1994), (Alvarez and de Prada, 1997), (Vada et al., 1999a), (Vada et al., 1999b) and (Vada et al., 1999c). To the best of the authors knowledge, the strategy presented in (Vada et $a l ., 1999 b)$ is the only optimal infeasibility handler which considers hard prioritized constraints without the use of a sequential solution approach. The focus in the present paper is on the application of this infeasibility handler. The usefulness of the method is illustrated on a simulated distillation column, and we present a novel stability result for this infeasibility handler combined with the Rawlings-Muske MPC controller (Rawlings and Muske, 1993).

The following notation is used throughout the paper: Let $n \geq 1$ be an integer and $x, y \in \mathbb{R}^{n}$. Then $\mathbb{I}_{n}^{+}:=\{1, \ldots, n\}, x \geq(>) y \Leftrightarrow x_{i} \geq(>) y_{i}$, $i \in \mathbb{I}_{n}^{+}$, and $0_{n}$ is an $n$-dimensional vector with zeros. $(x, y)$ is used to express $\left[x^{T}, y^{T}\right]^{T} . I_{n}$ is the $n \times n$ identity matrix.

\section{PROBLEM FORMULATION}

Let the system to be controlled be described by

$$
x_{t+1}=f\left(x_{t}, u_{t}, \eta_{t}\right),
$$

for some $f: \mathbb{R}^{n} \times \mathbb{R}^{m} \times \mathbb{R}^{n_{\eta}} \rightarrow \mathbb{R}^{n}$, where $x_{t}, u_{t}$, and $\eta_{t}$ denote the state-, control,- and disturbance-vector at time $t$ respectively. The presentation is based on the well known linear MPC problem (Rawlings and Muske, 1993): 


$$
\begin{array}{rlr}
\min _{\bar{\pi}_{t}} \phi\left(x_{t}, \bar{\pi}_{t}\right)=\sum_{j=t}^{\infty} x_{j \mid t}^{T} Q x_{j \mid t}+u_{j \mid t}^{T} R u_{j \mid t} \\
\text { subject to: } & \\
x_{t \mid t} & =x_{t} & \\
x_{t+N \mid t}^{u} & =0 & \\
x_{j+1 \mid t} & =A x_{j \mid t}+B u_{j \mid t}, & t \leq j \\
H x_{j \mid t} & \leq h, & t<j \leq j_{2}+t \\
D u_{j \mid t} & \leq d, & t \leq j \\
u_{j \mid t} & =0 & t+N \leq j
\end{array}
$$

where $Q \geq 0, R>0, \bar{\pi}_{t}=\left(u_{t \mid t}, \ldots, u_{t+N-1 \mid t}\right)$, $A \in \mathbb{R}^{n \times n}, B \in \mathbb{R}^{n \times m}$, and $x_{j \mid t} \in \mathbb{R}^{n}, u_{j \mid t} \in \mathbb{R}^{m}$, are the predicted state and control input vector at future time $j$, respectively, and $x_{j \mid t}^{u} \in \mathbb{R}^{n_{u}}$ denote the unstable modi of the predictor at future time $j$. The predictor is given by the third constraint in (2). Further, $H \in \mathbb{R}^{n_{h} \times n}, h>0 \in \mathbb{R}^{n_{h}}$, $D \in \mathbb{R}^{n_{d} \times m}$, and $d>0 \in \mathbb{R}^{n_{d}}$. Assume that $(A, B)$ is stabilizable and $N \geq \max \left\{n_{u}, 1\right\}$. Due to the constraint $u_{j \mid t}=0, t+N \leq j$, there exists a constraint horizon $j_{2} \geq N$ such that satisfaction of $H x_{j \mid t} \leq h, t<j \leq j_{2}+t$ implies $H x_{j \mid t} \leq h, t<j$ (Rawlings and Muske, 1993). Note that the system (1) and the predictor are equal if $f(x, u, \eta)=A x+B u$. This defines the nominal case.

The (linear) inequality constraints in (2) may be restated as

$$
S \bar{\pi}_{t} \leq s_{0}+S_{1} x_{t}
$$

where $S$ and $S_{1}$ are matrices, and $s_{0}$ is a vector. Note that the right hand side of (3) is parameterized by the state $x_{t}$. Thus, due to e.g. disturbances, operator interventions, or modelling errors, the state may take a value such that (3) has no solution. In the following we assume that $h$ and $d$ are constants. However, the proposed infeasibility handler can be used for time-varying $h$ and $d$ as well (operator intervention).

The constraints in an MPC optimization problem can be divided into the following classes:

Non-relaxable hard constraints: Hard inequality constraints that are absolute in the sense that they cannot under any circumstances be violated. Constraints related to physical limitations belongs to this class.

Relaxable hard constraints: Hard inequality constraints related to desirables. These constraints are relaxed only in cases when the whole set of hard constraints (i.e. relaxable and nonrelaxable) is inconsistent.

Soft constraints: Inequality constraints related to desirables. Violation of these constraints are allowed, but a term is included in the cost function (2) which penalizes constraint violations (see e.g. (Zheng and Morari, 1995), or (Scokaert and Rawlings, 1999)).

End point constraints: (I.e. $x_{t+N \mid t}^{u}=0$.) These are equality constraints related to stability. If these constraints are violated, nominal stability is not guaranteed (Rawlings and Muske, 1993).
Note that, generally, soft constraints, as opposed to relaxable hard constraints, can be violated even if this is not necessary in order to obtain a feasible solution to the original MPC optimization problem. In (Scokaert and Rawlings, 1999), the concept of exact soft constraints is introduced. This is a strategy to relax the soft constraints only when this is necessary in order to obtain a feasible solution of the MPC optimization problem. If this strategy is used, the soft constraints can be considered as relaxable hard constrains.

It is often the case that the constraints are not equally important, e.g. it is usually more important to satisfy the safety constraints than a product quality constraint. We divide the infeasibility handling strategies involving prioritization into two classes:

Hard prioritization: The prioritization among the constraints is absolute, i.e. a higher prioritized constraint is "infinitely" more important to fulfill than a lower prioritized constraint, see e.g. (Tyler and Morari, 1999), (Scokaert, 1994), (Vada et al., 1999a) and (Vada et al., 1999c).

Soft prioritization: The prioritization among the constraints is not absolute. This strategy is used in soft constrained MPC, where the individual weights on the different constraint violations in the penalty function determine the relative importance of each constraint. (See e.g. (Zheng and Morari, 1995), or (Scokaert and Rawlings, 1999)). Compared to hard prioritization, an advantage obtained by using soft prioritization is that it is straightforward to implement and it gives only a small increase in the on-line computational load. However, it is not straightforward to choose the weights so as to obtain the desired prioritization. In (Tyler and Morari, 1999) this problem is illustrated by an example. Such design difficulties is one of the reasons why we in the following concentrate on hard prioritization. Moreover, using hard prioritization, the relation between the specification and the achieved prioritization is explicit, and thus the design difficulties experienced by using soft prioritizations are not present. The on-line computational complexity of the approach we follow is comparable to the on-line computational complexity of a similar soft prioritization approach.

In the rest of this paper, unless otherwise stated, we make the following assumptions:

- There are no soft constraints.

- The MPC optimization problem is always feasible when all relaxable hard constraints are removed. (If this assumption is violated, some extraordinary action like shutdown is required).

- All necessary degrees of freedom are used to minimize the violation of the constraints, and all, if any, remaining degrees of freedom are used to minimize the cost function in (2). 


\section{OPTIMAL WEIGHT DESIGN PROBLEM}

In this section we formulate the problem of computing optimal constraint violations subject to hard prioritization as a single LP problem to be solved on-line at each sample. It is non-trivial to see that this is indeed possible, but this question was solved in (Vada et al., 1999b).

The constraints in the MPC optimization problem (2) can be transformed into the following three constraint sets:

$$
\begin{aligned}
G^{1} \pi_{t} & =g^{1}\left(x_{t}\right), g^{1}\left(x_{t}\right):=g^{10}+g^{11} x_{t} \\
G^{2} \pi_{t} & \leq g^{2}\left(x_{t}\right), g^{2}\left(x_{t}\right):=g^{20}+g^{21} x_{t} \\
G^{3} \pi_{t} & \leq g^{3}\left(x_{t}\right), g^{3}\left(x_{t}\right):=g^{30}+g^{31} x_{t} \\
\pi_{t} & \geq 0
\end{aligned}
$$

where $G^{1} \in \mathbb{R}^{n_{u} \times m \cdot N}, G^{2} \in \mathbb{R}^{m_{2} \times m \cdot N}, G^{3} \in$ $\mathbb{R}^{m_{3} \times m \cdot N}, g^{10} \in \mathbb{R}^{n_{u}}, g^{11} \in \mathbb{R}^{n_{u} \times n}, g^{20} \in \mathbb{R}^{m_{2}}$, $g^{11} \in \mathbb{R}^{m_{2} \times n}, g^{30} \in \mathbb{R}^{m_{3}}, g^{31} \in \mathbb{R}^{m_{3} \times n}$, and $\pi_{t}:=\bar{\pi}_{t}-\pi^{\text {min }} \in \mathbb{R}^{m \cdot N}$ is a modified vector of control inputs, where $\pi^{\mathrm{min}}$ is the lower limit on each control input. Such a limit will always be present in a practical MPC problem, since each element of $\bar{\pi}_{t}$ is related to a physical quantity. (However, if for some reason, $\pi_{i}^{\text {min }}$ does not exist, just replace $\pi_{i}$ with $u_{i}-v_{i}$ in (4), with $u_{i}^{\min }=$ $v_{i}^{\text {min }}=0$.) In $(4), G^{1} \pi_{t}=g^{1}\left(x_{t}\right)$ corresponds to the stability constraint $x_{t+N \mid t}^{u}=0$ in (2). Further, the inequality constraints in (2) are partitioned into the following two sets of constraints: $G^{2} \pi_{t} \leq$ $g^{2}\left(x_{t}\right)$, which is the set of all non-relaxable hard constraints, and $G^{3} \pi_{t} \leq g^{3}\left(x_{t}\right)$, which is the set of all relaxable hard constraints. The total number of inequality constraints in (2) is $n_{d} \cdot N+n_{h} \cdot j_{2}$, and thus $m_{2}+m_{3}=n_{d} \cdot N+n_{h} \cdot j_{2}$. The relation between (4) and (2) is easily established by, in (2), inserting the 1st, 3rd, and 6th constraint into the 2 nd and 4 th constraint and by replacing $\bar{\pi}_{t}$ with $\pi_{t}+\pi^{\mathrm{min}}$. This is detailed in (Vada et al., $1999 b$ ). Further, assume that there exists a hard prioritization among the inequalities in $G^{3} \pi_{t} \leq$ $g^{3}\left(x_{t}\right)$, and that $G^{3}$ and $g^{3}$ are constructed such that the $i$ th row of $G^{3} \pi_{t} \leq g^{3}\left(x_{t}\right)$ have higher priority than the $(i+1)$ th row. This implies that minimizing the violations of the $i$ th row of $G^{3} \pi_{t} \leq g^{3}\left(x_{t}\right)$ is "infinitely" more important than minimizing the violations of the $(i+1)$ th row.

Assume that, at a given sample, the optimization problem in (2) is infeasible, that is, there is no feasible solution to (4). Since the 3 rd constraint in (4) is the only relaxable hard constraint, in order to transform (2) into a feasible optimization problem, we introduce a vector of constraint violations $z_{t} \in \mathbb{R}^{m_{3}}$ as follows

$$
\begin{aligned}
& G^{1} \pi_{t}=g^{1}\left(x_{t}\right) \\
& G^{2} \pi_{t} \leq g^{2}\left(x_{t}\right) \\
& G^{3} \pi_{t} \leq g^{3}\left(x_{t}\right)+z_{t} \\
& \pi_{t}, z_{t} \geq 0
\end{aligned}
$$

Next we introduce the notion of lexicographic minimum: $y^{o} \in Y \subseteq \mathbb{R}^{n}$ is the lexicographic minimum of $Y$ if it is not possible to find another $y \in Y$ and an $i \in \mathbb{I}_{n}^{+}$such that $y_{i}<y_{i}^{o}$ and $y_{j}=$ $y_{j}^{o}, j \in \mathbb{I}_{i-1}^{+}$. As an example $[0.10,0.01,10000]$ is lexicographically less than $[0.10,0.011,0]$, since the first element of both vectors are equal, while minimizing the second element is "infinitely" more important than minimizing the third.

Now we are ready to state a problem whose solution can be used to compute optimal constraint violations (according to the given hard prioritization) by solving only one LP problem on-line in addition to the original MPC QP problem:

Optimal weight design problem (OWDP)

Let $X \neq \emptyset$ denote the set of all $x_{t}$ such that there exists $\left(\pi_{t}, z_{t}\right)$ satisfying (5). Given an $x_{t} \in X$, let $Z\left(x_{t}\right)$ denote the set of all $z_{t} \geq 0$ such that there exists a $\pi_{t}$ satisfying the inequalities (5). Design the weight vector $\tilde{c}$ in (6) such that $\forall x_{t} \in X, z_{t}^{*}$ defined by

$$
\left(\pi_{t}^{*}, z_{t}^{*}\right):=\operatorname{argmin} \tilde{c}^{T} z \quad \text { subject to (5), }
$$

is equal to the lexicographic minimum of $Z\left(x_{t}\right)$.

Note that in the OWDP, for a given $x_{t}, z_{t}^{*}$, the optimum of (6), represents the optimal constraint violations of the constraints in (2) with respect to the given hard prioritization. That is, there does not exist any $z_{t} \in Z\left(x_{t}\right)$ which violates these constraints less with respect to the given hard prioritization. Also note that since we have assumed that $d, h>0,(A, B)$ stabilizable, and $N \geq \max \left\{n_{u}, 1\right\}$, we have that $X \neq \emptyset$ and $0 \in \operatorname{int} X$. In (Vada et al., 1999b), existence of a solution to the OWDP under these assumptions is established, and in (Vada et al., 1999c) it is shown how the OWDP may be solved. A consequence of this result is: At each sample, if $i$ ) the state $x_{t}$ has a value making (2) infeasible, and $i i) x_{t} \in X$, that is, with the given $x_{t}$ there exists a relaxation of the relaxable hard constraints such that (2) becomes feasible, then an optimal relaxation $z_{t}^{*}$ can be computed by solving the LP problem in (6).

\section{STABILITY}

In this section we show that by combining the proposed infeasibility handler with the MPC controller defined in Section 2, the region of attraction of the original MPC controller without an infeasibility handler is increased. For a certain prioritization, Theorem 1 below establishes nominal asymptotic stability for the receding horizon implementation of (2) if the constraints in (2) are replaced by (5) with $z_{t}=z_{t}^{*}$, where the weights $\tilde{c}$ in (6) is a solution to the OWDP. First, we present Lemma 1 which is needed in the proof of Theorem 1.

Lemma 1

Assume that the constraints $x_{t+j \mid t} \in \tilde{X}, \forall j>1$, are hard non-relaxable constraints in (2), where 
$\tilde{X} \subset \mathbb{R}^{n}, 0 \in \operatorname{int} \tilde{X}$, is an arbitrary bounded subset of $X$. Then, in (2), there exists a sufficiently large $j_{2} \geq N$ such that $\forall x_{t} \in \tilde{X}, H x_{t+j_{2} \mid t} \leq h \Rightarrow$ $H x_{t+j_{2}+i \mid t} \leq h, i=1,2, \ldots$.

The lemma follows from (Rawlings and Muske, 1993) and boundedness of $\tilde{X}$. Next, we define a prioritization among the constraints which is used in Theorem 1:

\section{Priority Assumption}

Assume that a unique priority level is assigned to each relaxable row of $H x_{j \mid t} \leq h$ and $D u_{j \mid t} \leq d$ in (2), such that all constraints on the horizon related to a certain relaxable row of $H x_{j \mid t} \leq h$ or $D u_{j \mid t} \leq d$ with a given priority level have higher priority than any constraint on the horizon related to rows with a lower priority level . Let $H_{i}$ $\left(D_{i}\right)$ denote the ith row of $H(D)$, and assume that $\forall j \in \mathbb{I}_{j_{2}-1}^{+}, i \in \mathbb{I}_{n_{h}}^{+}, t \geq 0, H_{i} x_{t+j+1 \mid t} \leq h_{i}$ has higher priority than $H_{i} x_{t+j \mid t} \leq h_{i}$, and that $\forall j \in \mathbb{I}_{N}^{+}, i \in \mathbb{I}_{n_{d}}^{+}, t \geq 0, D_{i} u_{t+j \mid t} \leq h_{i}$ has higher priority than $D_{i} u_{t+j-1 \mid t} \leq d_{i}$.

\section{Theorem 1}

Assume that the constraints $x_{t+j \mid t} \in \tilde{X}, \forall j>1$ are hard non-relaxable constraints in (2), and let $j_{2}$ be given as in Lemma 1. Let $G^{3}$ and $g^{3}$ in (5) be constructed according to the Priority Assumption. Assume further that i) $f(x, u, \eta)=A x+B u$, ii) $\forall t \geq 0, z_{t}=z_{t}^{*}$, the solution to (6), where $\tilde{c}$ is a solution to the $O W D P$, and iii) $\forall t \geq 0, u_{t}=u_{t \mid t}^{*}$, where $u_{t \mid t}^{*}$ is the first $m$ elements of the solution of (2) where the constraints are replaced by (5). Then, $\forall x_{0} \in \tilde{X},\left\{z_{t}\right\}_{t=0}^{\infty}$ becomes zero within finite time, and the origin is an asymptotically stable equilibrium point with $\tilde{X}$ contained in the region of attraction.

Proof. First we prove that $z_{t}^{*}$ becomes zero in finite time: Given any $x_{0} \in \tilde{X}$, let $z_{0}^{\prime}$ denote the constraint violations obtained by shifting the constraint violations in $z_{0}^{*}$ one step ahead and filling up with zeros in the locations corresponding to prediction $j_{2}+1 \mid 1(N \mid 1)$ for the state (control input) constraints. Thus, since $x_{1}=x_{1 \mid 0}$ (nominal case), at time $t=1, z_{1}=z_{0}^{\prime}$ is feasible, and hence zero violation of the constraint at the end of the horizon of the highest prioritized constraint is feasible. Then, due to the choice of $\tilde{c}$ in (6), the corresponding element of $z_{1}^{*}$ is equal to zero. Continuing this argument, due to the prioritization along the horizon, we obtain that in $z_{t}^{*}$, all violations of $H x_{j \mid t} \leq h$ (or $D u_{j \mid t} \leq d$ ) corresponding to the highest priority level, becomes zero after at least $j_{2}$ (or $N$ ) samples. Continuing this for the row of $H x_{j \mid t} \leq h$ or $D u_{j \mid t} \leq d$ corresponding with the next priority level, and so on, we obtain that $z_{t}^{*}=0_{m_{3}}, t=m_{3}, m_{3}+1, \ldots$.

Finally we prove asymptotic stability with $\tilde{X}$ contained in the region of attraction: Let $X^{\prime}$ be the set of all $x_{t}$ such that there exists a

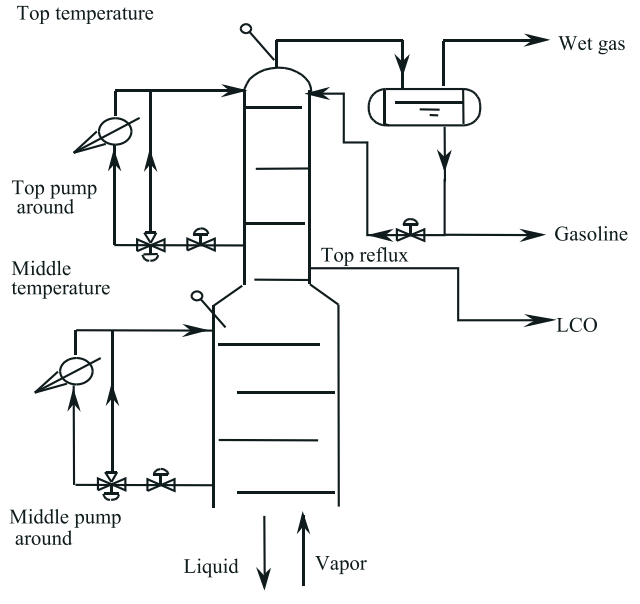

Fig. 1. Top part of a FCCU fractionator.

$\pi_{t}$ satisfying (5) with $z_{t}=0$. It follows from (Rawlings and Muske, 1993) that $\forall x \in X^{\prime}$, by using the control law defined by receding-horizon implementation of the solution of (2), the origin is an asymptotically stable solution. Combining this with the fact that $z_{t}^{*}=0_{m_{3}}, t=m_{3}, m_{3}+1, \ldots$, the result follows.

Note that we need to assume that $\forall t \geq 0, x_{t}$ is contained in a bounded region $\tilde{X}$. This is done to obtain a fixed $j_{2}$ which is sufficiently large to be valid for all $x_{t} \in \tilde{X}$. Also note that a result similar to Theorem 1 is stated in (Rawlings and Muske, 1993) and (Scokaert and Rawlings, 1999) for the case when only the state constraints can be relaxed, and when all rows of $H x_{j \mid t}$ have equal priority. An important consequence of Theorem 1 is that by using the proposed controller, the region of attraction of the MPC controller (2) without infeasibility handling is enlarged from $X^{\prime}$ to $\tilde{X}$ (cf. the proof of Theorem 1 for $X^{\prime}$ ).

\section{SIMULATION EXAMPLE}

In this section, we illustrate the use of the proposed infeasibility handler for a linear model of the top section of a fluid catalytic cracker unit (FCCU) main fractionator, see Figure 1, which is a critical unit for separating gasoline and LCO (diesel) from the feedstock from an upstream riser reactor. A rigorous model of the fractionator has been developed and fitted to real plant data (Cong et al., 1998), and a linear model has been derived by linearization of this model around a nominal operating point:

$$
x_{t+1}=A x_{t}+B u_{t},
$$

where

$$
\begin{aligned}
& A=0.01\left[\begin{array}{rrrr}
90.9028 & -6.4632 & 5.7545 & 1.5616 \\
-1.2803 & 92.1012 & 1.5499 & 3.3564 \\
-0.7713 & -4.2529 & 85.3340 & 3.4314 \\
0.2413 & -0.7122 & -0.1415 & 85.6537
\end{array}\right], \\
& B=0.01\left[\begin{array}{rrrrr}
-3.6674 & -12.4077 & -0.0648 & -5.7626 & 0.1049 \\
-2.0582 & -0.5071 & 0.1088 & -7.2823 & 0.2324 \\
-1.3759 & -1.6691 & 1.5177 & -1.8955 & -0.1224 \\
0.1182 & -0.0777 & -0.0002 & -0.3235 & 1.4456
\end{array}\right],
\end{aligned}
$$


Table 1. States and control inputs.

\begin{tabular}{|l|l|l|}
\hline Var. & Description & Unit \\
\hline \hline$\left(x_{t}\right)_{1}$ & Top vapor temperature. & ${ }^{\circ} \mathrm{C}$ \\
\hline$\left(x_{t}\right)_{2}$ & Middle vapor temperature. & ${ }^{\circ} \mathrm{C}$ \\
\hline$\left(x_{t}\right)_{3}$ & Top heat exchanger outlet temperature. & ${ }^{\circ} \mathrm{C}$ \\
\hline$\left(x_{t}\right)_{4}$ & Middle heat exchanger outlet temp. & ${ }^{\circ} \mathrm{C}$ \\
\hline$\left(u_{t}\right)_{1}$ & Top pump-around tee valve position & $\%$ \\
\hline$\left(u_{t}\right)_{2}$ & Top reflux valve position. & $\%$ \\
\hline$\left(u_{t}\right)_{3}$ & Top pump-around valve position. & $\%$ \\
\hline$\left(u_{t}\right)_{4}$ & Middle pump-around tee valve position. & $\%$ \\
\hline$\left(u_{t}\right)_{5}$ & Middle pump-around valve position. & $\%$ \\
\hline
\end{tabular}

Table 2. Operating point and bounds.

\begin{tabular}{|l|l||l|l||ll|}
\hline$x_{1}^{\text {nom }}$ & $107.0{ }^{\circ} \mathrm{C}$ & $x_{1}^{a b s, l b}$ & 106 & $x_{1}^{a b s, u b}$ & 108 \\
\hline$x_{2}^{\text {nom }}$ & $219.0{ }^{\circ} \mathrm{C}$ & $x_{2}^{a b s, l b}$ & 218 & $x_{2}^{a b s, u b}$ & 221 \\
\hline$x_{3}^{\text {nom }}$ & $87.0{ }^{\circ} \mathrm{C}$ & $x_{3}^{a b s, l b}$ & 83 & $x_{3}^{a b s, u b}$ & 91 \\
\hline$x_{4}^{\text {nom }}$ & $199.0{ }^{\circ} \mathrm{C}$ & $x_{4}^{a b s, l b}$ & 195 & $x_{4}^{a b s, u b}$ & 203 \\
\hline$u_{1}^{\text {nom }}$ & $62.3 \%$ & $u_{1}^{a b s, l b}$ & 0 & $u_{1}^{a b s, u b}$ & 100 \\
\hline$u_{2}^{\text {nom }}$ & $0 \%$ & $u_{2}^{a b s, l b}$ & 0 & $u_{2}^{a b s, u b}$ & 100 \\
\hline$u_{3}^{\text {nom }}$ & $50 \%$ & $u_{3}^{a b s, l b}$ & 40 & $u_{3}^{a b s, u b}$ & 80 \\
\hline$u_{4}^{\text {nom }}$ & $67.9 \%$ & $u_{4}^{a b s, l b}$ & 0 & $u_{4}^{a b s, u b}$ & 100 \\
\hline$u_{5}^{\text {nom }}$ & $50 \%$ & $u_{5}^{a b s, l b}$ & 40 & $u_{5}^{a b s, u b}$ & 80 \\
\hline
\end{tabular}

Table 3. Relaxable hard constraints.

\begin{tabular}{|c|c||c|c|}
\hline Pri. level & Constraint & Pri. level & Constraint \\
\hline \hline 1 & $\left(x_{t}^{a b s}\right)_{1} \leq 107.5$ & 6 & $\left(u_{t}^{a b s}\right)_{3} \leq 55$ \\
\hline 2 & $\left(x_{t}^{a b s}\right)_{2} \leq 219.5$ & 7 & $\left(u_{t}^{a b s}\right)_{5} \leq 55$ \\
\hline 3 & $\left(x_{t}^{a b s}\right)_{1} \geq 106.5$ & 8 & $\left(u_{t}^{a b s}\right)_{1} \leq 67$ \\
\hline 4 & $\left(x_{t}^{a b s}\right)_{2} \geq 218.5$ & 9 & $\left(u_{t}^{a b s}\right)_{4} \leq 75$ \\
\hline 5 & $\left(u_{t}^{a b s}\right)_{2} \leq 5.0$ & & \\
\hline
\end{tabular}

and $x_{t}:=x_{t}^{a b s}-x^{n o m}$, and $u_{t}:=u_{t}^{a b s}-u^{n o m}$, where $x^{\text {nom }}:=\left(x_{1}^{\text {nom }}, \ldots, x_{4}^{\text {nom }}\right)$ and $u^{\text {nom }}:=$ $\left(u_{1}^{\text {nom }}, \ldots, u_{5}^{\text {nom }}\right) .\left(x^{\text {nom }}, u^{\text {nom }}\right)$ is the nominal operating point. The sampling time is $30 \mathrm{~s}$. The legend for the states and control inputs is given in Table 1, and the nominal operating point in addition to the absolute upper and lower bounds are given in Table 2. Non-relaxable hard constraints are defined as $x^{a b s, l b} \leq x_{t}+x^{n o m} \leq x^{a b s, u b}$, and $u^{a b s, l b} \leq u_{t}+u^{n o m} \leq u^{a b s, u b}$. Further, the relaxable hard constraints and their corresponding priority levels are given in Table 3 . The prioritizations are based on assumptions such as gasoline is assumed to be more valuable than LCO. Note that since the constraint horizon in (2) is $j_{2}$, there are $j_{2}$ constraints corresponding to each of the above defined state constraints (both relaxable and non-relaxable), and that due to the move horizon, there are $N$ constraints corresponding to each of the above defined control input constraints (both relaxable and non-relaxable). Hence, there are several constraints related to a given priority level. Assume the same prioritization as in the Priority Assumption (defined in Section 4). We have chosen $N=5$, and by using a slight modification of (Gilbert and Tan, 1991, Algorithm 3.2) to calculate $j_{2}$, assuming that the non-relaxable hard state constraints are always satisfied, we get $j_{2}=40$. Thus, for the given example, there
Table 4 . Weights $\tilde{c}_{i}$ which are different from 1.0.

\begin{tabular}{|c|c|c|}
\hline$i$ & $\tilde{c}_{i}$ & Corresponds to: \\
\hline \hline 79 & 25.70 & $\left(x_{t+2 \mid t}^{a b s}\right)_{2} \leq 219.5$ \\
\hline 80 & 113.9 & $\left(x_{t+1 \mid t}^{a b s}\right)_{2} \leq 219.5$ \\
\hline 119 & 14.86 & $\left(x_{t+2 \mid t}^{a b s}\right)_{1} \geq 106.5$ \\
\hline 120 & 62.91 & $\left(x_{t+1 \mid t}^{a b s}\right)_{1} \geq 106.5$ \\
\hline 165 & 1.070 & $\left(u_{t \mid t}^{a b s}\right)_{2} \leq 5$ \\
\hline 180 & 1.283 & $\left(u_{t \mid t}^{a b s}\right)_{1} \leq 67$ \\
\hline
\end{tabular}

are $m_{3}=185$ distinct priority levels, and in the OWDP, the dimension of $\tilde{c}$ is thus 185 .

In order to solve the OWDP we used Algorithm 4.4 in (Vada et al., 1999c). In the algorithm, the parameter determining the lower bound on the weights is set equal to 1.0. The elements of the resulting $\tilde{c}$ which are greater than 1.0 are shown in Table 4.

The algorithm is implemented in MATLAB with NAG Foundation Toolbox, and the computation time was about 76 minutes on a Pentium $450 \mathrm{MHz}$ PC with $256 \mathrm{MB}$ RAM. Note, however, that the computation of $\tilde{c}$ is done off-line. The on-line computational effort associated with the infeasibility handler (the LP problem in (6)) is typically smaller than the QP (2).

Note that only six of the weights are greater than their minimum value. At a first glance, since the weights related to the 78 highest prioritized constraints are all equal to the predetermined minimum value, one might think that it is remarkable that these weights solve the OWDP. However, note that all weights in Table 4 are related to the first or second samples on the horizon for a given priority level. Thus, for the given process, minimizing the constraint violations at the beginning of the horizon implies that the constraint violations at the end of the horizon are minimized. (Recall that within a given priority level, the constraints corresponding to the first samples of the horizon have lower priority than the samples at the end of the horizon.) Note that this might not be the case for a different process. Further note that it is by far not intuitive to determine how large the weights should to be in order to guarantee the fulfillment of the hard prioritization. The largest weight produced by this algorithm is only two orders of magnitude larger than the smallest weights. This is in strong contrast to a heuristic approach that might rely on using a sufficiently large weight ratio between each priority level. The latter approach could lead to a numerically illconditioned LP problem.

The simulation result obtain by combining the proposed infeasibility handler with the closed-loop implementation of (2) when a state disturbance of $[-1,2,-4,4]^{T}$ enters the system at $t=0$ is shown in Figure 2. In (2), $Q=100 I_{n}$ and 

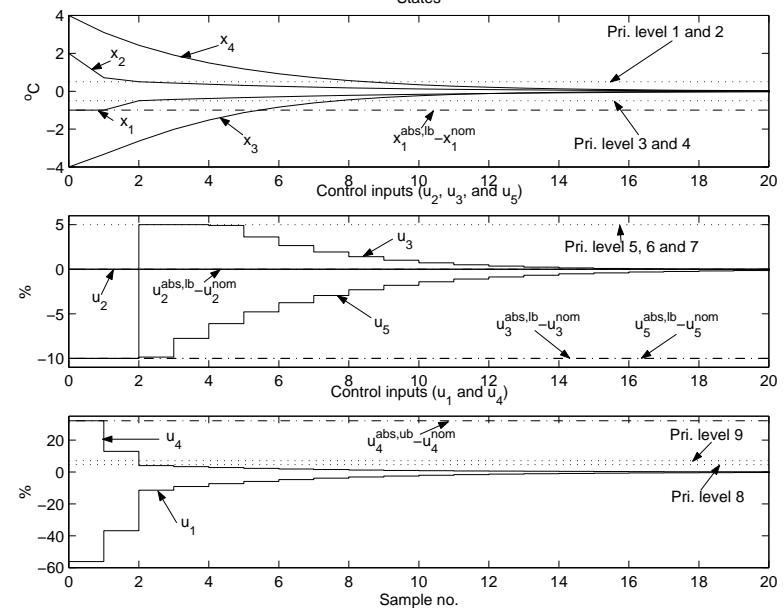

Fig. 2. Simulation results. All values are deviations from the nominal operating point. Solid lines: states and control inputs, dash-dotted lines: non-relaxable constraints, dotted lines: relaxable constraints. Note that in the upper part, only the hard constraint on $x_{1}$ are shown, since the other hard constraints are active only at $t=0$.

$R=I_{m}$. Observe that all relaxable constraints are satisfied for all $t \geq 2$. At $t=0$, there are 4 relaxable constraints which are violated. Two of them corresponds to the first sample of the constraints with priority level 2 and 3 , and the other two corresponds to the first two samples of the constraint with priority level 9 . At $t=1$, the only constraint violation corresponds to the first sample of the constraint with priority level 9 .

\section{DISCUSSION/CONCLUSIONS}

Whether or not the assumption of hard prioritization is realistic, is dependent on the application.

The calculations in Section 5 establishes the practical viability of the proposed infeasibility handler, i.e that, for the present example, the off-line computational load is not prohibitive large, and that the resulting optimization problem (an LP) to be solved at each sample is not ill-conditioned.

Traditionally, when designing constraints which are desirables (not related to physical limitations), one needs to consider whether or not such constraints may cause the controller to run into feasibility problems. By using the proposed approach for infeasibility handling, such considerations become less important. Actually, one might design relaxable hard constraints which one knows can be satisfied in only small regions of the state space.

The paper also proves that the proposed strategy guarantees nominal asymptotic stability if avoiding constraint violations at the end of the horizon has the highest priority. This result implies that the region of attraction of the controller without infeasibility handling is enlarged by using the proposed infeasibility handler.

\section{REFERENCES}

Alvarez, T. and C. de Prada (1997). Handling Infeasibilities in Predictive Control. Computers chem. Engng. 21, 577-582.

Cong, S. B., P. Yuan and F. Shen (1998). An integrated non-equilibrium dynamic model of petroleum distillation column. Unpublished manuscript.

Garcia, C. E. and A. M. Morshedi (1986). Quadratic Programming Solution of Dynamic Matrix Control (QDMC). Chemical Engineering Communications 46, 73-87.

Gilbert, E. G. and K. T. Tan (1991). Linear Systems with State and Control Constraints: The Theory and Application of Maximal Output Admissible Sets. IEEE Transactions on Automatic Control 36(19), 1008-1020.

Qin, S. J. and T. A. Badgwell (1996). An Overview of Industrial Model Predictive Control Technology. In: Proceedings of Chemical Process Control - CPC V. AIChE. Tahoe City, CA.

Rawlings, J B. and K R. Muske (1993). The Stability of Constrained Receding Horizon Control. IEEE Transactions on Automatic Control 38(10), 1512-16.

Scokaert, P. O. M. (1994). Constrained predictive control. PhD thesis. University of Oxford, UK.

Scokaert, P. O. M. and J. B. Rawlings (1999). Feasibility issues in model predictive control. AIChE Journal 45(8), 1649-59.

Tyler, M. L. and M. Morari (1999). Propositional logic in control and monitoring problems. Automatica 35, 565-582.

Vada, J., O. Slupphaug and B. A. Foss (1999a). Infeasibility Handling in Linear MPC subject to Prioritized Constraints. In: Preprints of 14th World Congress of IFAC, Beijing, China. Vol. N. pp. 163-168.

Vada, J., O. Slupphaug and T. A. Johansen $(1999 b)$. Efficient Infeasibility Handling in Linear MPC subject to Prioritized Constraints. In: Proceedings of European Control Conference '99. European Union Control Association. Karlsruhe, Germany. Paper ID: F395.

Vada, J., O. Slupphaug and T. A. Johansen (1999c). Efficient Optimal Prioritized Infeasibility Handling in Model Predictive Control - a Parametric Preemptive Multi-Objective Linear Programming Approach. Submitted to Journal of Optimization Theory and Applications.

Zheng, A. and M. Morari (1995). Stability of Model Predictive Control with Mixed Constraints. IEEE Transactions on Automatic Control 40(10), 1818-23. 